\title{
Impact of Various Desensitizing Agents on Occlusion of Dentinal Tubules: A Scanning Electron Microscopic Study
}

\author{
Arti Dixit ${ }^{1}$, Naman Awasthi $^{2}$, Anupama Ahirwar $^{3}$, Tarun Nanu ${ }^{4}, \mathrm{~K}$ Binu Nathan ${ }^{5}$, Jeslee A Jose ${ }^{6}$
}

\begin{abstract}
Aim: The aim of the present study was to compare the capacity of three dissimilar commercially accessible desensitizing products for the occlusion of dentinal tubules using a scanning electron microscope (SEM).

Materials and methods: The nondiseased human mandibular premolar teeth, ninety in number, were amassed for this study. The blocks subsequently made were subjected to polishing using abrasive paper to facilitate dentinal surface exposure. The samples were randomly allocated to one of the following three groups: Group I, samples to be treated with Admira Protect; Group II, samples to be treated with MI paste, Group III, samples to be treated with Remin Pro. Photomicrograph of every sample was taken to evaluate the occlusion of the dentin tubule under 2000× magnification of the SEM. Results: MI paste exhibited the greatest dentin tubular occlusion with a value of $2.746 \pm 0.530$ followed by Admira Protect (3.498 \pm 0.202$)$ and Remin Pro $(4.594 \pm 0.364)$ in that order. Amid the various desensitizing materials used, statistically significant differences $(p<0.001)$ were noted.

Conclusion: Within the confines of the limitations of the present research, following a comparative assessment of the three desensitizing materials used, it can be concluded that all of them were efficient in sealing off the dentin tubules in spite of differing chemical constitutions and techniques of application. When compared with the agents, Admira Protect and Remin Pro, MI paste exhibited the maximum occluding capacity of the dentinal tubules.

Clinical significance: A short, sharp shooting pain characterizes the frequent problem of dentin hypersensitivity (DH). Sealing off the open dentin tubules, thereby reducing dentinal permeability, is a known method in the treatment of $\mathrm{DH}$. As several agents exist to reduce $\mathrm{DH}$, the best commercially marketed product for decreasing this condition by occluding the tubules should be identified by the clinicians.

Keywords: Dentin hypersensitivity, Dentinal tubule, Desensitizing agents, Scanning electron microscope.

The Journal of Contemporary Dental Practice (2021): 10.5005/jp-journals-10024-3129
\end{abstract}

\section{INTRODUCTION}

Dentinal sensitivity has prevalence between $8 \%$ and $57 \%$ globally and is a frequent problem in adults. Individuals with exposed and permeable dentin owing to loss of the tooth surface mainly experience pain. Pain is usually a result of provocation of the dentine that has been exposed and stimulation of dentinal tubules by the nerves in the pulp from various external factors such as chemical, tactile, evaporative, thermal, osmotic, and mechanical agents that are not attributable to any type of dental deformities or pathologies. When affected, the patients present with sharp, spontaneous, and transient pain of short duration following provocation by a stimulus. This is then followed by deep, dull aching pain. Pain may be generalized when affecting various surfaces of the teeth or can be localized to a small number of the teeth.

The hydrodynamic theory justifies dentine hypersensitivity (DH) in all probability. According to this theory, pain in cases of $\mathrm{DH}$ is a result of alterations in fluid flow contained in the exposed dentine tubules. Decreasing the permeability of the dentin and reducing the response of intradental nerves to shifts in fluid have hence been suggested as the two methods for dentin desensitization. ${ }^{2}$

$\mathrm{DH}$ peaks between the second and the fourth decade of life especially in the buccocervical area of the teeth owing to the dentin tubule branching at the dentinoenamel junction (DEJ). The capability of desensitizing agents to close or occlude the tubules of dentine and decrease the open tubule diameter to reduce pain transmitted by the hydrodynamic mechanism is a direct measure of the success of agents used in desensitization. To aid in extended pain relief, the agents used in desensitization must be able to
${ }^{1}$ Department of Public Health Dentistry, Vaidik Dental College and Research Centre, Daman, Daman and Diu, India

${ }^{2}$ Department of Dentistry, Government Medical College, Shahdol, Madhya Pradesh, India

${ }^{3}$ Department of Dentistry, Gandhi Medical College, Bhopal, Madhya Pradesh, India

4,5Department of Conservative Dentistry and Endodontics, Educare Institute of Dental Sciences, Malappuram, Kerala, India

${ }^{6}$ Department of Conservative Dentistry and Endodontics, Yenepoya Dental College, Mangaluru, Karnataka, India

Corresponding Author: Arti Dixit, Department of Public Health Dentistry, Vaidik Dental College and Research Centre, Daman, Daman and Diu, India, Phone: +91 9712042464, e-mail:dr.dixitarti@gmail.com

How to cite this article: Dixit A, Awasthi N, Ahirwar A, et al. Impact of Various Desensitizing Agents on Occlusion of Dentinal Tubules: A Scanning Electron Microscopic Study. J Contemp Dent Pract 2021;22(7):829-832.

Source of support: Nil

Conflict of interest: None

endure the dynamic oral atmosphere with the tests of dissolution by saliva, microbial acid attack, and constituents of food in addition to other thermal, chemical, and mechanical trauma. ${ }^{3}$

Gingival recession is notably the most frequent cause of exposure of the dentinal tubules, and $\mathrm{DH}$ is strongly linked to the dentine tubules that are exposed. Some causes of DH include chewing of tobacco, dietary, and brushing habits as well as certain 
diseases such as gastroesophageal reflux disease. DH may also result from wasting disorders such as abrasion, abfraction, erosion, and root surface denudation or could be the result of surgical and nonsurgical periodontal therapy. ${ }^{4}$

Several marketed products decrease $\mathrm{DH}$ by lessening permeability such as fluoride; zinc chloride; hydroxyapatite; potassium chloride; strontium chloride; dental adhesives, bioglass, portland cement, glass ionomer cement, oxalates, and casein phosphopeptide-amorphous calcium phosphate (CPP-ACP); and laser treatment. $^{5}$

As occlusion of the dentinal tubules with chemical materials is a feasible therapeutic intervention, recognition of the preeminent commercially available compound for decreasing the pain by occluding the tubules should be identified. Hence, this study was undertaken with an aim to compare the capacity of three dissimilar commercially accessible desensitizing products for occlusion of dentinal tubules.

\section{Materials and Methods}

The present in vitro study was conducted in the Department of Public Health Dentistry, Vaidik Dental College and Research Centre, Daman, India. Nondiseased human mandibular premolar teeth, ninety in number, which had been extracted (18 to 24 years of age) during the orthodontic treatment process, were amassed for this study. Exclusion criteria included samples with coronal or root surface dental caries, presence of restoration, cracked/ fractured teeth, history of pulpal or periapical pathosis, and dentinal sclerosis. The specimens were thoroughly cleansed, and 5\% sodium hypochlorite solution was used disinfection for 1 hour. The teeth were then stored in distilled water.

Plastic blocks $30 \times 30 \times 30 \mathrm{~mm}^{3}$ were collected that were filled with plaster of Paris. Subsequently, the specimen teeth were put into the plaster such that just the buccal surfaces were not covered to facilitate preparation of cavities on the cervical region of each surface.

Inverted-cone bur (Dia-bur, Zhengzhou, Henan, China) was used to prepare cavities $2 \mathrm{~mm}$ deep and $3 \mathrm{~mm}$ wide on cervical regions buccally. The attained samples were subjected to polishing using an abrasive paper (600-1200 grit, SS White, Gloucester, England) to facilitate dentinal surface exposure. To ensure complete opening of the dentin tubules, the blocks were immersed in $17 \%$ ethylenediaminetetraacetic acid for 40 minutes. Blocks were kept in for 40 minutes to completely open the dentinal tubules. Ultrasonication of the blocks in distilled water for 12 minutes was performed to get rid of the residual smear layer.

The sample teeth were washed using distilled water and dried with air. They were then placed in a hot air oven at $37^{\circ} \mathrm{C}$ for 2 hours and examined under a scanning electron microscope (SEM) to scrutinize the dentinal tubular opening that would replicate hypersensitive dentin in the cervical region. After the analysis under SEM (Zeiss sigma VP, Zeiss Oberkochen, Germany) with 2000x magnification, the baseline dentinal tubular opening values were noted. Then, the samples were subjected to washing and then preserved in artificial saliva for 24 hours. The composition of the saliva substitute was sodium carboxymethylcellulose $(10.0 \mathrm{gm})$, $\mathrm{KCl}(0.625 \mathrm{gm}), \mathrm{K}_{2} \mathrm{HPO}_{4}(0.804 \mathrm{gm}), \mathrm{CaCl}_{2} \cdot 2 \mathrm{H}_{2} \mathrm{O}(0.166 \mathrm{gm})$, $\mathrm{MgCl}_{2} \cdot 6 \mathrm{H}_{2} \mathrm{O}(0.059 \mathrm{gm})$, methyl-p-hydroxybenzoate (2.0 gm), and $\mathrm{KH}_{2} \mathrm{PO}_{4}(0.326 \mathrm{gm})$ in 1 liter of water.

The samples were randomly allocated to one of the following three groups:
Group I: Samples were treated with Admira Protect: Admira Protect (VOCO America Inc., Indian land, South Carolina, USA) was used to treat all the samples in this group. In accordance with the manufacturer's instructions, the application of Admira Protect was performed using a fine application brush such as Micro Tim in an even manner on all the dentinal surfaces. It was permitted to act for a period of 20 seconds and then subjected to light-curing, and the application were performed twice daily (with 12 hours of interval) for 7 days.

Group II: Samples were treated with the MI paste: The samples in this group were applied with the MI paste (GC, Melbourne, Australia) agent twice daily (with 12 hours of interval for 3 minutes) for 7 days. The samples were thereafter kept in artificial saliva after every period of treatment.

Group III: Samples were treated with Remin Pro: The samples in this group received Remin Pro (VOCO, Cuxhaven, Germany) on their dentinal surfaces using a disposable brush twice daily (with 12 hours of interval for 3 minutes) for 7 days.

\section{Analysis of Dentinal Tubules Occlusion Using an SEM}

The sample teeth that were preserved in artificial saliva were washed under distilled water and dried with air. They were then placed in a hot air oven at $37^{\circ} \mathrm{C}$ for 2 hours. After this, the samples were preserved in a vacuum desiccator for additional 2 hours, and then they were sputter coated to help conductivity. Photomicrograph of every sample was taken to evaluate occlusion of the dentin tubule under $2000 \times$ magnification of the SEM (Zeiss sigma VP, Zeiss Oberkochen, Germany).

\section{SEM Scoring Criteria}

Two observers independently performed blinded assessment to document tubular occlusion. An assessment was carried out in accordance with the tubule occlusion classification scoring system as below ${ }^{6}$ :

- Score 1-Occluded (100\% of the tubules closed);

- Score 2-Mostly occluded (50\% to less than $100 \%$ of the tubules closed);

- Score 3-Partially occluded (25\% to less than $50 \%$ of the tubules closed);

- Score 4-Mostly unoccluded (less than 25\% of the tubules closed);

- Score 5-Unoccluded (0\%, no tubular closure observed).

The mean score of the dentin tubule closure as evaluated by both the blinded observers was taken and used for the analysis.

\section{Statistical Analysis}

Statistical analysis of the data procured was performed with one way analysis of variance and Tukey's post hoc test. SPSS version 20 was used for the analysis (SPSS Inc.,Chicago, Illinois, USA). A value of 0.05 was set as the probability level of statistical significance.

\section{Results}

The mean scores of the dentin tubular occlusion after the application of the desensitizing products are shown in Table 1. MI paste exhibited the greatest dentin tubular occlusion with a value of $2.746 \pm 0.530$ followed by Admira Protect $(3.498 \pm 0.202)$ and Remin Pro $(4.594 \pm 0.364)$. Amid the various desensitizing materials used, statistically significant differences $(p<0.001)$ were noted. 
Table 1: Comparison of the mean score of dentinal tubule occlusion after applying desensitizing agents

\begin{tabular}{lcccc}
\hline Desensitizing agents & Mean $\pm S D$ & Std. error & $F$ & pvalue \\
\hline Group I: Admira Protect & $3.498 \pm 0.202$ & 0.0169 & & \\
Group II: MI paste & $2.746 \pm 0.530$ & 0.0214 & 9.610 & $0.001^{*}$ \\
Group III: Remin Pro & $4.594 \pm 0.364$ & 0.0138 & & \\
\hline
\end{tabular}

"Statistically significant

Table 2 depicts results from the Turkey test which indicate that the difference between Admira Protect and Remin Pro as well as amid MI paste and Remin Pro groups of desensitizing products used were statistically significant. The difference between Admira Protect and MI paste was not statistically significant.

\section{Discussion}

Short-duration severe pain may be experienced by the dentin and is referred to as DH. This is commonly caused by acidic corrosion, mechanical wear, and abrasion. The foundation for conduction of these painful sensations is the movement of the fluid in the tubular interstitium. A likely way of decreasing or completely abating these symptoms of pain due to $\mathrm{DH}$ is intercepting conduction of stimuli to neural ends of the odontoblastic processes by decreasing movement of fluid within tubules by sealing off or decreasing the tubular openings. Occlusion within orifices, surface, and subsurface dentin sealing of the dentinal tubules can be accomplished. Intradentinal occlusion is thought to be the most capable approach in regard to the success of long duration. ${ }^{7}$

Following the large existing acceptance of the Brannstrom's hydrodynamic theory, materials that proficiently occlude dentinal tubules have been used as desensitizing agents in dental clinics.

Vieira and Santiago ${ }^{8}$ and Pashley et al. ${ }^{9}$ conducted lab studies to assess the effects of agents used in desensitization on sealing of dentinal tubules by comparison of variations in permeability before and after the application of the desensitizer on a dentin disk linked with a capillary in a chamber that was split. Although this methodology was useful owing to the ease of sample preparation and application of the desensitizing material, the majority of the research exerted a greater pressure when compared with the physiological pulp pressure to hasten air bubble motion in the flow measuring device capillary.

In the present study, the greatest sealing of dentin tubules was noted in MI paste, followed by Admira Protect and Remin Pro group in that order. The MI paste is composed of CPP-ACP along with sodium fluoride at a concentration of $0.2 \%$. CPP stands for a casein phosphopeptide that is able to stabilize amorphous calcium phosphate which is insoluble as the CPP-ACP complex. ${ }^{10} \mathrm{MI}$ paste is known to cause remineralization by depositing calcium phosphate precipitates composed of fluoride, which aids in prevention of $\mathrm{DH}$ and dental caries. The studies carried out by Tang et al. ${ }^{11}$ and Martinez-Mier ${ }^{12}$ show that there was no significant sealing of dentin tubules using MI Paste.

A study conducted by Soares et al. ${ }^{13}$ stated that the remineralization of tissues occurs owing to the presence of $900 \mathrm{ppm}$ of fluoride in CPP-ACP. It also exhibited effective decrease in the $\mathrm{DH}$ on follow-up which is attributable to raised bioavailability of calcium and phosphate concentrations. Added to this is the synergism of fluoride as a constituent along with such ions, that incorporates them in the dental biofilm resulting in stable mineral
Table 2: Multiple comparison of mean score of dentinal tubule occlusion after applying desensitizing agents using Tukey's post hoc test

\begin{tabular}{llcc}
\hline Groups & Compared with & Mean difference & Significance \\
\hline Admira Protect & Ml paste & 0.752 & 0.082 \\
& Remin Pro & -1.096 & $0.001^{*}$ \\
\multirow{3}{*}{ Ml paste } & Admira Protect & -0.752 & 0.082 \\
& Remin Pro & -1.848 & $0.001^{*}$ \\
\multirow{2}{*}{ Remin Pro } & Admira Protect & 1.096 & $0.001^{*}$ \\
& Ml paste & 1.848 & $0.001^{*}$ \\
\hline
\end{tabular}

"Statistically significant

precipitates containing fluorapatite with resultant decrease in sensitivity.

Admira Protect in the present study exhibited higher sealing of the dentin tubules as compared with the Remin Pro group. 2-hydroxyethyl methacrylate monomers, organic acids, and ormocers as well as bisphenol A diglycidyl ether dimethacrylate are the chief constituents of Admira Protect. Inorganic silanated filler particles and inorganic-organic copolymers constitute ormocer substances. Manufacturers state that they exhibit dentin bonding similar to self-etching adhesives. ${ }^{14}$ Torres et al. ${ }^{15}$ and Pereira et al. ${ }^{16}$ have cited that this need not comprise chemical agents required for polymerization. Fluid movement reduction could be a result of proteins precipitated within the dentinal tubules.

Despite this, numerous limitations of this method still exist and have been declared. The penetration depth of materials in the dentin tubules has not been approximately noted as increase in penetration would aid in long-lasting pain relief arising from $\mathrm{DH}$. The mechanism by which these substances act in sealing the dentin tubules has also not been analyzed. Two blinded observers only did a surface examination of the dentin tubules after application of the desensitizers. Future long-term research and comparative clinical-based studies with certain other ways of desensitization must be performed to validate its supremacy.

\section{Conclusion}

Within the confines of the limitations of the present research, following a comparative assessment of the three desensitizing materials used, it can be concluded that all of them were efficient in sealing off the dentin tubules in spite of differing chemical constitutions and techniques of application. When compared with the agents, Admira Protect and Remin Pro, MI paste exhibited the maximum occluding capacity of the dentinal tubules.

\section{References}

1. Markowitz K, Pashley DH. Discovering new treatments for sensitive teeth: the long path from biology to therapy. J Oral Rehabil 2008;35(4):300-315. DOI: 10.1111/j.1365-2842.2007.01798.x.

2. Wang Z, Sa Y, Sauro S, et al. Effect of desensitising toothpastes on dentinal tubule occlusion: a dentine permeability measurement and SEM in vitro study. J Dent 2010;38(5):400-410. DOI: 10.1016/ j.jdent.2010.01.007.

3. Mohammed Wasim Bari L, Sunil Kumar S, Datta Prasad S, et al. Scanning electron microscopic evaluation of the effectiveness of desensitizing agents on dentinal tubule occlusion and durability - an in vitro study. Acta Sci Dent Sci 2019;3(7):57-62. DOI: 10.31080/ASDS.2019.03.0570.

4. Pinto SC, Pochapski MT, Wambier DS, et al. In vitro and in vivo analysis of desensitizing agents on dentin permeability and 
dentin tubule occlusion. J Oral Sci 2010;52(1):23-32. DOI: 10.2334/ josnusd.52.23.

5. Ghafournia $M$, Tehrani $M H$, Nekouei $A$, et al. In vitro evaluation of dentin tubule occlusion by three bioactive materials: a scanning electron microscopic study. Dent Res J (Isfahan) 2019;16(3):166-171. https://pubmed.ncbi.nlm.nih.gov/31040872/.

6. Chen $\mathrm{CL}$, Parolia A, Pau A, et al. Comparative evaluation of the effectiveness of desensitizing agents in dentine tubule occlusion using scanning electron microscopy. Aust Dent J 2015;60(1):65-72. DOI: 10.1111/adj.12275.

7. Paes Leme AF, dos Santos JC, Giannini M, et al. Occlusion of dentin tubules by desensitizing agents. Am J Dent 2004;17(5):368-372. https://pubmed.ncbi.nlm.nih.gov/15575450/.

8. Vieira AH, Santiago SL. Management of dentinal hypersensitivity. Gen Dent 2009;57(2):120-126. https://pubmed.ncbi.nlm.nih. gov/19552361/.

9. Pashley DH, Stewart FP, Galloway SE. Effects of air drying in vitro on human dentine permeability. Arch Oral Biol 1984;29(5):379-383. DOI: 10.1016/0003-9969(84)90164-x.

10. Li Y, Lee S, Mateo LR, et al. Comparison of clinical efficacy of three professionally applied pastes on immediate and sustained reduction of dentinhypersensitivity. Compendium2013;34(1):e6-e12. https:// www.aegisdentalnetwork.com/cced/2013/01/.
11. Tang B, Millar BJ. Effect of chewing gum on tooth sensitivity following whitening. Br Dent J 2010;208(12):571-577. DOI: 10.1038/ sj.bdj.2010.500.

12. Martinez-Mier EA. Casein phosphopeptide used in toothpaste suggests an efficacy similar to toothpaste containing sodium monofluorophosphate for caries prevention. J Evid Based Dent Pract 2010;10(3):154-155. DOI: 10.1016/j.jebdp.2010.06.003.

13. Soares R, De Ataide IDN, Fernandes M, et al. Assessment of enamel remineralisation after treatment with four different remineralising agents: a Scanning Electron Microscopy (SEM) study. J Clin diagnostic Res 2017;11(4):ZC136-ZC141. DOI: 10.7860/ JCDR/2017/23594.9758.

14. Malkoç MA, Sevimay M. Evaluation of mineral content of dentin treated with desensitizing agents and neodymium yttriumaluminium-garnet (Nd: YAG) laser. Lasers Med Sci 2012;27(4):743-748. DOI: 10.1007/s10103-011-0954-6.

15. Torres CR, Silva TM, Fonseca BM, et al. The effect of three desensitizing agents on dentin hypersensitivity: a randomized, split-mouth clinical trial. Oper Dent 2014;39(5):E186-E194. DOI: 10.2341/13-057.

16. Pereira JC, Martineli AC, Tung MS. Replica of human dentin treated with different desensitizing agents: a methodological SEM study in vitro. Braz Dent J 2002;13(2):75-85. DOI: 10.1590/s010364402002000200001. 